\section{Characterization of off-axis fishbones}

To cite this article: W W Heidbrink et al 2011 Plasma Phys. Control. Fusion 53085028

View the article online for updates and enhancements.

\section{Related content}

-'Beam-emission spectroscopy' diagnostics also measure edge fast-ion light

- Beam-ion confinement for different injection geometries

- Central flattening of the fast-ion profile in reversed-shear DIII-D discharges

\section{Recent citations}

- Mechanisms of energetic-particle transport
$\frac{\text { in magnetically confined plasmas }}{\text { W. W. Heidbrink and R. B. White }}$
- Origin of ion cyclotron emission at the
$\frac{\text { proton cyclotron frequency from the core }}{\text { of deuterium plasmas in the ASDEX- }}$
$\frac{\text { Upgrade tokamak }}{\text { B Chapman et al }}$
- Comparing theory and simulation of ion
$\frac{\text { cyclotron emission from energetic ion }}{\text { populations with spherical shell and ring- }}$
$\frac{\text { beam distributions in velocity-space }}{\text { B Chapman et al }}$




\title{
Characterization of off-axis fishbones
}

\author{
W W Heidbrink ${ }^{1}$, M E Austin ${ }^{2}$, R K Fisher ${ }^{3}$, M García-Muñoz ${ }^{4}$, \\ G Matsunaga $^{5}$, G R McKee ${ }^{6}$, R A Moyer $^{7}$, C M Muscatello ${ }^{1}$, \\ M Okabayashi $^{8}$, D C Pace ${ }^{9}$, K Shinohara ${ }^{5}$, W M Solomon ${ }^{8}$, E J Strait ${ }^{3}$, \\ M A Van Zeeland ${ }^{3}$ and Y B Zhu ${ }^{1}$ \\ ${ }^{1}$ Department of Physics and Astronomy, University of California, Irvine, CA, USA \\ ${ }^{2}$ Institute of Fusion Studies, University of Texas at Austin, Austin, TX, USA \\ ${ }^{3}$ General Atomics, San Diego, CA, USA \\ ${ }^{4}$ Max-Planck-Institut für Plasmaphysik, Garching, Germany \\ 5 Japan Atomic Energy Agency, Naka City, Ibaraki, Japan \\ ${ }^{6}$ Department of Engineering Physics, University of Wisconsin at Madison, Madison, WI, USA \\ ${ }^{7}$ Center for Energy Research, University of California, San Diego, La Jolla, CA, USA \\ ${ }^{8}$ Princeton Plasma Physics Laboratory, Princeton, NJ, USA \\ ${ }^{9}$ Oak Ridge Institute for Science and Education, Oak Ridge, TN, USA
}

Received 28 February 2011, in final form 13 June 2011

Published 7 July 2011

Online at stacks.iop.org/PPCF/53/085028

\begin{abstract}
Repetitive bursting instabilities with strong frequency chirping occur in highbeta, beam-heated plasmas with safety factor $q>1$ in the DIII-D tokamak. Although the mode structures differ, in many ways, the off-axis fishbones are similar to the $q=1$ fishbones first observed on the Poloidal Divertor Experiment (PDX). The modes are driven by energetic trapped ions at the fastion precession frequency. During a burst, the frequency changes most rapidly as the mode reaches its maximum amplitude. Larger amplitude bursts have larger growth rates and frequency chirps. Unlike PDX fishbones, the decay phase is highly variable and is usually shorter than the growth phase. Also, the waveform is highly distorted by higher harmonics during the latter portion of a burst. The radial mode structure alters its shape during the burst. Like PDX fishbones, the modes expel trapped ions in a 'beacon' with a definite phase relationship relative to the mode. Seven types of loss detectors measure the beacon. The losses scale linearly with mode amplitude. The neutron rate changes most rapidly at maximum mode amplitude but, depending on the loss diagnostic, the losses often peak a few cycles later. The non-ambipolar fast-ion losses cause a sudden change in toroidal rotation frequency across the entire plasma. In addition to an overall drop, the neutron signal oscillates in response to the wave. Unlike the beacon of lost particles, which maintains a fixed phase relative to the mode, the phase of the neutron oscillations steadily increases throughout the burst, with the greatest phase slippage occurring in the highly nonlinear phase near maximum mode amplitude.
\end{abstract}

(Some figures in this article are in colour only in the electronic version) 


\section{Introduction}

The fishbone instability was first observed [1] during deuterium near-perpendicular neutral beam injection into the Poloidal Divertor Experiment $(\mathrm{PDX})$. The mode had an $(m, n)=(1,1)$ structure in the plasma, as expected for an internal kink. (Here $m$ and $n$ denote the poloidal and toroidal mode numbers, respectively.) The fishbones occurred in periodic bursts. During each burst, the mode frequency chirped down in frequency from (typically) 20 to $12 \mathrm{kHz}$. The neutron emission dropped at each burst, with the rate of change peaking near the time of peak mode amplitude [2]. Neutral particle analyzers (NPAs) detected a 'beacon' of expelled trapped fast ions, a burst of signal with a fixed phase relative to the magnetic fluctuation on each mode cycle $[3,4]$. The transport of fast ions was explained as convective radial transport caused by resonance between the fast-ion precession motion and the kink mode [5]. The instability was identified as a new branch of the internal kink associated with the large energetic ion population [6].

Subsequently, $q=1$ fishbone instabilities were observed on many other devices. (A comprehensive review of both the PDX fishbones and subsequent measurements on other tokamaks, including DIII-D, appears in [7].) Both an MHD branch [8] and an energeticparticle branch are theoretically predicted [9] and observed [7]. A comprehensive picture emerged: at modest fast-ion density, the trapped ions help stabilize the fluid branch but, at large fast-ion density, they destabilize the energetic-particle branch [10].

Fishbone-like modes in plasmas with central safety factor well above unity were first observed on JET [11]. More recently, fishbone-like modes were studied in JT-60U [12,13] and DIII-D [14, 15] plasmas with similar $q$ profiles as the JET plasmas but higher beta. While $q=1$ fishbones have eigenfunctions whose maximum is inside the $q=1$ surface, the mode structure for the off-axis fishbones peaks near the $q=2$ surface $[11,15]$. Some authors have suggested that the off-axis fishbones are internal kinks [11], while others suggest that they are related to an external kink [15]. In either case, it is likely that the off-axis fishbone is an energetic-particle branch of the fluid internal or external kink mode. A theoretical study showed that a modest fast-ion population can stabilize an internal double kink mode but a large population destabilizes a new fishbone branch [16], just as it does for the $1 / 1$ internal kink. On the other hand, the observation that the instabilities occur in plasmas that are susceptible to resistive wall modes suggests that the off-axis fishbones may be an energetic-particle branch of the MHD external kink [15]. (Resistive wall modes occur in plasmas that would be unstable to external kinks in the absence of a conducting wall [17].) A recent paper [15] shows that the JT-60U and DIII-D modes have the same phenomenology and discusses their relationship to the resistive wall mode.

The goal of this paper is to provide a detailed description of the off-axis fishbone in DIII-D. The data are compared and contrasted to PDX fishbones throughout the paper. The nonlinear evolution of the off-axis fishbone bursts displays novel features not previously reported for any fast-ion driven instability.

The paper begins with a description of the plasma conditions and diagnostics (section 2). Next, the mode is characterized (section 3.1) and the loss (section 3.2) and confined (section 3.3) fast-ion data are described. Speculation about the reasons for the differences between PDX fishbones and off-axis fishbones appears in section 4, together with the conclusion.

\section{Apparatus}

All of the data in this paper are from DIII-D. Most of the discharges described in this paper were discussed in the DIII-D portion of the paper by Okabayashi et al [15]. A database of 513 bursts 


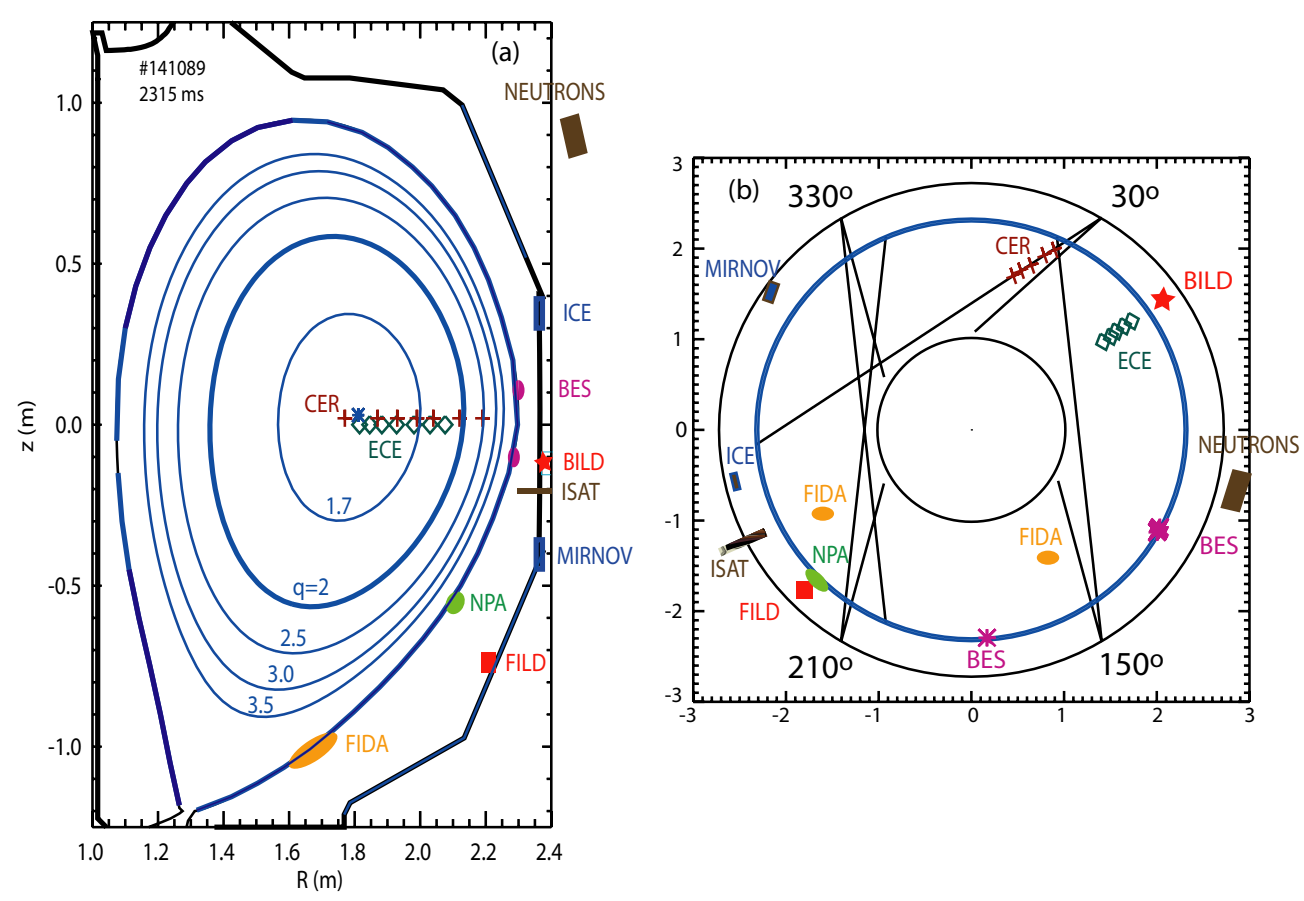

Figure 1. (a) Elevation of the DIII-D vacuum vessel, showing the locations of the various measurements. (The BES, NPA and FIDA signals depend on the edge neutral density profile, so the indicated positions are estimates.) The flux surfaces for a typical equilibrium are labeled by their $q$ value; $q_{0}=1.5$ on axis and $q_{95}=4.4$. (b) Plan view. The lines represent the center of the beamlines. The toroidal field (plasma current) is in the clockwise (counter-clockwise) direction.

was compiled that contains all of the $n=1$ off-axis fishbone bursts observed during two days of operation. The plasma conditions are similar for nearly all of the bursts: plasma current $I_{\mathrm{p}}=0.95-1.14 \mathrm{MA}$; toroidal field $B_{\mathrm{T}}=1.72$; minor radius $a=0.60 \mathrm{~m}$; normalized beta $\beta_{\mathrm{N}}=\beta_{\mathrm{T}} /\left(I_{\mathrm{p}} / a B_{\mathrm{T}}\right)=2.1-2.7$; line-average plasma density $\bar{n}_{\mathrm{e}}=(3.4-4.2) \times 10^{19} \mathrm{~m}^{-3}$. The beam power was the actuator for feedback control of $\beta_{\mathrm{N}}$, so it varies between 5.7 and $14.9 \mathrm{MW}$ for the bursts in the database, with a typical average power of $10 \mathrm{MW}$. The beams inject both in the co- $I_{\mathrm{p}}$ direction $(\leqslant 15 \mathrm{MW})$ and in the counter- $I_{\mathrm{p}}$ direction $(\leqslant 5 \mathrm{MW})$; both neartangential (tangency radius $\left.R_{\tan }=1.15 \mathrm{~m}\right)$ and near-perpendicular $\left(R_{\tan }=0.76 \mathrm{~m}\right)$ angles are employed. The average injection energies for the co-tangential, co-perpendicular, countertangential, and counter-perpendicular beams are $66-80 \mathrm{keV}, 60-73 \mathrm{keV}, 80-81 \mathrm{keV}$ and 60 $75 \mathrm{keV}$, respectively. Typically, the beam beta is $\sim 1 / 6$ of the total beta. In many discharges, $\sim 2.7 \mathrm{MW}$ of electron cyclotron current drive power is injected to avoid neoclassical tearing modes [14]. The plasma is deuterium, the neutral beams inject deuterium atoms, and the primary impurity is carbon from the graphite walls $\left(Z_{\text {eff }} \simeq 2\right)$.

All of the discharges have the shape shown in figure 1 , a lower single null divertor configuration. The $\nabla B$ drift is downward toward the divertor and all of the plasmas are in H-mode. The equilibrium is based on EFIT [18] solutions of the Grad-Shafranov equation that use motional Stark effect [19] measurements of the internal magnetic field. The central safety factor is $q_{0}=1.15-1.78$, the magnetic shear is relatively weak near the magnetic axis, crosses the $q=2$ surface near a major radius of $R=2.1 \mathrm{~m}$ and rises rapidly to a value of $q_{95}=4.3-4.7$ at the edge. $\left(q_{95}\right.$ is the safety factor at the surface that encloses $95 \%$ of the poloidal flux.) Typical central electron and ion temperatures are $T_{\mathrm{e}}=5$ and $T_{\mathrm{i}}=6 \mathrm{keV}$. 
Figure 1 shows the locations of the principal diagnostics. Toroidal and poloidal arrays of Mirnov coils [20] are the primary fluctuation diagnostic. In this paper, all of the Mirnov coil traces are from a coil that is located at a toroidal angle of $307^{\circ}$. At this magnetic field and density, the electron cyclotron emission (ECE) diagnostic [21] measures optically thick, third harmonic ECE emission for the spatial positions shown in the figure. The charge-exchange recombination (CER) diagnostic [22] measures the temperature, toroidal rotation, and density of carbon ions; on some discharges, the CCD camera acquired data in $0.5 \mathrm{~ms}$ time bins to detect rapid changes in toroidal rotation.

The primary diagnostic for the confined fast ions is a neutron scintillator that measures $2.5 \mathrm{MeV}$ neutrons produced in deuterium-deuterium fusion reactions. Two complementary scintillators with identical electronics are mounted beside a vacuum flange just outside the vacuum vessel: a plastic scintillator and a $\mathrm{ZnS}\left({ }^{6} \mathrm{Li}\right)$ scintillator. The plastic scintillator can resolve oscillations in neutron flux with frequencies $\gg 10 \mathrm{kHz}$, while the $\mathrm{ZnS}$ scintillator has an effective bandwidth of $\sim 3 \mathrm{kHz}$ [23]. Consistent with the expected instrumental response, the fluctuations in neutron flux discussed in section 3.3 are much weaker on the $\mathrm{ZnS}$ signal than on the plastic signal. All neutron data in this paper are from the plastic scintillator.

Seven different diagnostics detect escaping fast ions. The beam-ion loss detector (BILD) is a Faraday cup that is mounted on the edge of a vacuum port $12 \mathrm{~cm}$ below the midplane [24]; in this paper, the signals are from the foil that detects co-circulating orbits. The fast-ion loss detector (FILD) is a scintillator-based diagnostic that resolves the gyroradius and pitch of the escaping fast ions in an image that is recorded on a CCD camera [25]. For higher bandwidth measurements, a photomultiplier detects light from a portion of the scintillator. A reciprocating Langmuir probe with robust graphite tips [26] measures fluctuations in ion saturation current (ISAT); at the time of the measurements, the probe was in the scrape-off region $6.1 \mathrm{~cm}$ from the last-closed flux surface and $5.6 \mathrm{~cm}$ from the outer wall. A solid-state NPA operated in current mode measures fluctuations in charge-exchange reactions when fast ions escape to the high neutral density region at the plasma edge. The fast-ion D-alpha (FIDA) diagnostic [27, 28] measures Doppler-shifted light emitted by fast ions after they undergo a charge-exchange reaction. For the rapidly changing background conditions associated with these fishbone bursts, the temporal resolution of the spectroscopic FIDA diagnostics proved inadequate to detect changes in core fast-ion density; however, bursts of edge FIDA emission are observed by the filter-based f-FIDA diagnostic [28] on both 'active' sightlines that view an injected beam and 'passive' sightlines that do not intersect an injected beam. The beam-emission spectroscopy (BES) diagnostic [29] suffers from contamination by edge FIDA light for these fishbone bursts [30], complicating use of the data for internal eigenfunction measurements of density fluctuations. However, for one discharge where the neutral beams viewed by the BES diagnostic were off, the signals are entirely from passive FIDA light produced when fast ions are expelled to the high neutral density region at the plasma edge [30]. The seventh edge loss diagnostic is indirect. When fast ions are expelled into the scrape-off region, they usually destabilize ion cyclotron emission (ICE) [7]. These waves are thought to be magnetoacoustic waves excited by the anisotropic 'bump-on-tail' distribution function caused by the losses [31]. For these experiments, the signal from a high-bandwidth toroidal coil [32] was filtered by two bandpass filters, rectified and digitized. The 'lower pass' filter includes the first deuterium cyclotron harmonic at the plasma edge, while the 'higher pass' filter spans the second and third harmonics.

All fishbone bursts that meet certain criteria are included in the database. Figure 2 shows examples of included and excluded bursts. Like the first burst in figure 2(a), all of the included bursts are $n=1$ modes that rotate in the direction of the plasma current. Roughly $3 \%$ of the 

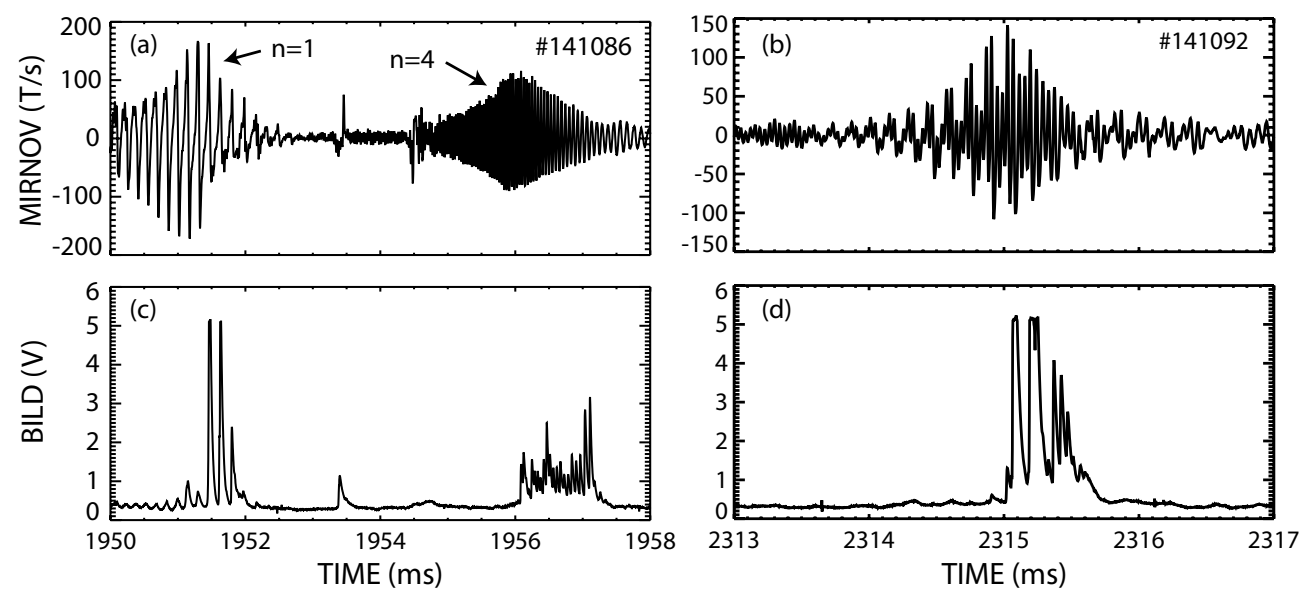

Figure 2. Sample Mirnov coil $(a),(b)$ and BILD $(c),(d)$ signals for bursts that are excluded from the database. (a), (c) The $n=1$ burst at $1951 \mathrm{~ms}$ in $(a)$ is included but the $n=4$ burst at $1957 \mathrm{~ms}$ is excluded. $(b),(d)$ Bursts with appreciable beating due to a steady tearing mode (in this case, an $n=3$ mode) are also excluded. Note that the BILD signals saturate at $5 \mathrm{~V}$.

observed bursts have higher toroidal mode numbers (usually $n=3$ or $n=4$ ). Like the $n=1$ modes, these higher frequency modes chirp down in frequency and cause losses that have a definite phase relative to the wave; however, these higher frequency modes are not discussed further here. There are also many examples of fishbone bursts that occur in plasmas with steady tearing modes (figure $2(b)$ ). Phenomenologically, these bursts appear identical to the retained bursts but, because of difficulties in accurately analyzing the Mirnov coil signal in the presence of strong beating, they are excluded from our database.

\section{Data}

\subsection{Mode properties}

The off-axis fishbone bursts typically last for $2-3 \mathrm{~ms}$ and occur about every $20 \mathrm{~ms}$. Figure 3 shows a typical Mirnov coil signal. Initially, the signal is nearly sinusoidal during the growth phase. The amplitude grows approximately exponentially. At maximum amplitude, the waveform becomes strongly distorted from a sine wave. Data from the toroidal array show that this distortion is associated with the appearance of higher $n$ harmonics: in other words, the entire structure becomes distorted. The mode period visibly lengthens as the burst passes through its maximum amplitude.

Figure 3 also illustrates analysis of the signal for inclusion in the database. First, the maxima and minima are identified (figure $3(a)$ ). Then the rectified amplitude of the maxima and minima are plotted (figure $3(b)$ ). Note that, during the rise phase, the amplitudes of the maxima and minima have nearly the same value but, during the decay phase, the maxima are larger than the minima. The amplitude data are fit to exponentials to obtain the growth and decay rates. In the third step, the time difference between the maxima and minima is graphed (figure $3(c)$ ). The initial mode frequency is obtained from a fit to the first several cycles, while the final mode frequency is obtained from the last two cycles. The frequency chirping rate is obtained from a linear fit to the change in period near peak mode amplitude. The mode distortion is derived from the difference in period between half-cycles, $\Delta T_{1 / 2} / \bar{T}_{1 / 2}$. 

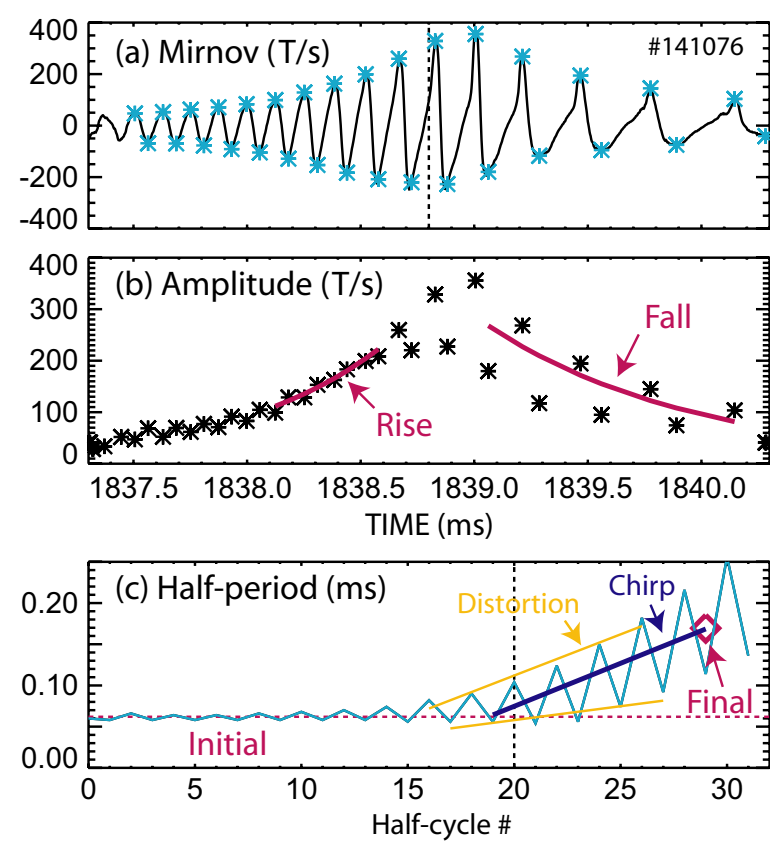

Figure 3. (a) Mirnov coil signal for a typical fishbone burst. The symbols indicate the maxima and minima used for calculation of the half-period. (b) Amplitude of the maxima and minima for the same burst, together with exponential fits to the growth and decay phases. (c) Half-period in milliseconds for each half-cycle throughout the burst. The initial frequency is derived from a horizontal fit at low amplitude. The frequency chirping rate is obtained from a linear fit near maximum amplitude. (The dashed vertical line indicates the time of maximum amplitude.) The final frequency is an average over the last two cycles. Linear fits to the long half-periods and the short half-periods are used to measure the waveform distortion.

(This quantity is zero for a sine wave.) This definition of distortion gives similar results to alternative definitions [15] based on the amplitude or the deviation from a sine wave. The rate of change of the distortion is also recorded in the database.

The observed rise time increases with the maximum amplitude of the mode (figure 4(a)). The correlation coefficient $r$ is 0.72 for these data. (Figure 4(a) shows the correlation with $\mathrm{d} \tilde{B} / \mathrm{d} t$; the correlation with the peak amplitude $\tilde{B}$ is slightly higher $(r=0.76)$.) PDX fishbones had a similar rise time and dependence on mode amplitude [33]. In contrast, as shown in figure $4(b)$, the decay rate is uncorrelated with mode amplitude $(r=0.05)$. Unlike PDX fishbones, the decay rate is generally faster than the growth rate. An example of the wide variation in behavior during the decay phase for a pair of otherwise similar bursts is shown in figure 5. This example illustrates the following general features: (1) the evolution of the bursts is quite reproducible during the growth phase; (2) frequency chirping and mode distortion always occur near maximum amplitude; (3) the decay phase is highly variable. Sometimes the burst decays in a few cycles, while other times it persists for many. Often the mode distortion remains large but sometimes nearly sinusoidal successor oscillations persist at small amplitude following the primary, highly distorted, crash.

The magnitude of the frequency chirping scales with mode amplitude (figure $4(c)$ ) $(r=0.77)$. The rate of change of the chirping rate also scales with mode amplitude $(r=0.55)$. The frequency chirping also increased with mode amplitude for PDX fishbones [2]. Within a given burst, the chirping rate is largest in the period just after maximum amplitude. Similarly, 

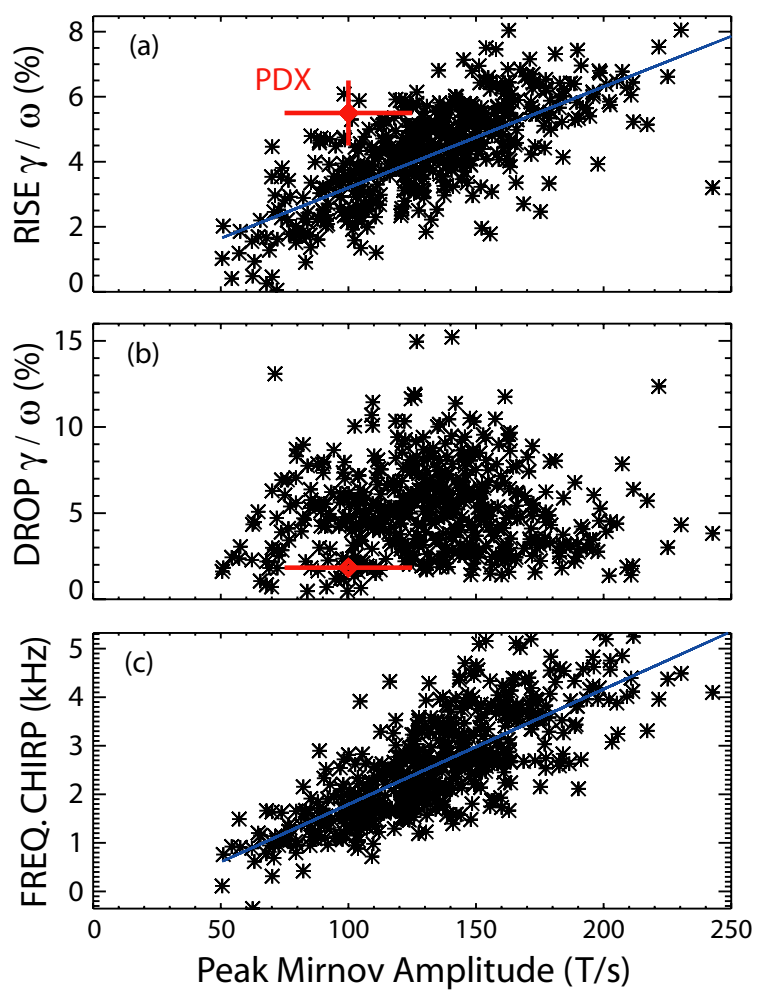

Figure 4. (a) Growth rate, $(b)$ decay rate and $(c)$ change in frequency $f_{\mathrm{i}}-f_{\text {final }}$ versus peak mode amplitude for all of the bursts in the database. The growth and decay rates are normalized to the mode frequency in the plasma frame, $\omega=2 \pi\left(f_{\mathrm{i}}-f_{\text {rot }}\right)$, where $f_{\text {rot }}$ is from the $R=212 \mathrm{~cm}$ CER channel. The lines are linear fits to the data. The error bars are the values observed for typical cases in PDX [33].

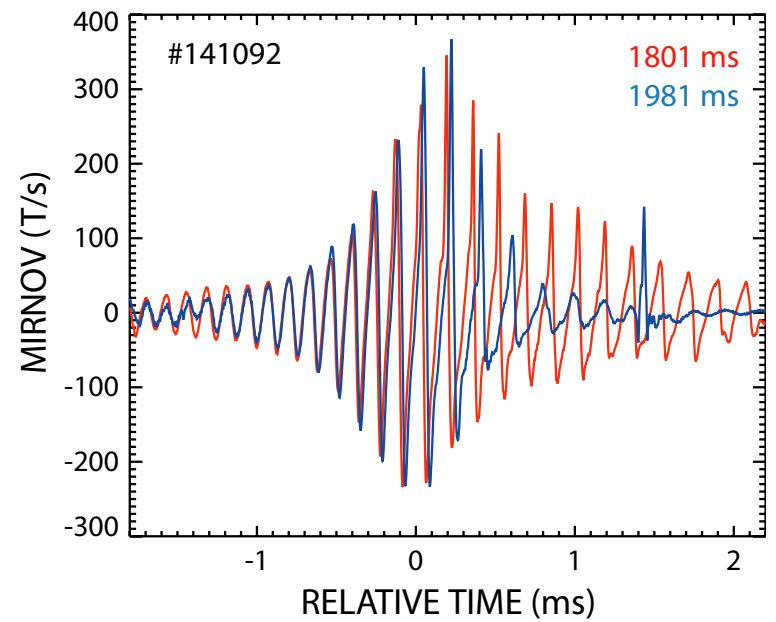

Figure 5. Example of two similar bursts with strikingly different behavior in the decay phase. 


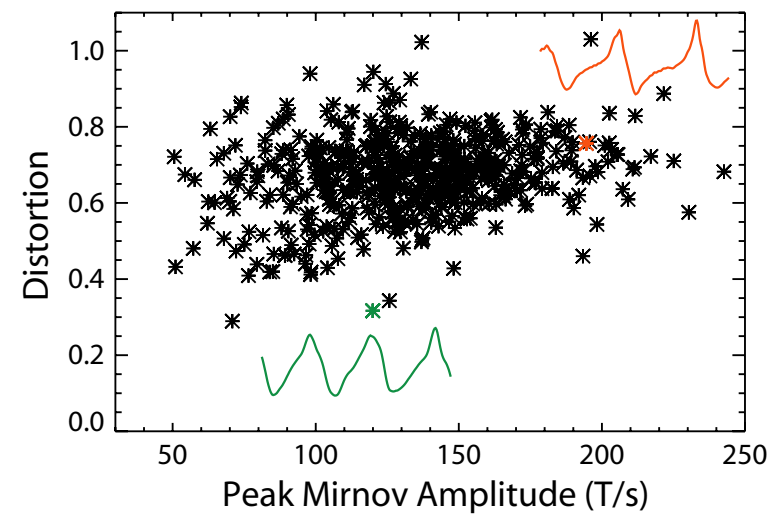

Figure 6. Distortion of the waveform $\Delta T_{1 / 2} / \bar{T}_{1 / 2}$ near maximum mode amplitude versus peak Mirnov amplitude for all of the bursts in the database. Illustrative examples of waveforms with distortions of 0.32 and 0.76 are included.

PDX fishbones [33] and high frequency chirping instabilities in DIII-D [34] chirp the fastest near maximum mode amplitude.

All of the fishbones exhibit mode distortion (figure 6). Most bursts exhibit very strong distortion but even the most weakly distorted waveforms deviate significantly from a sine wave. Analysis of the poloidal Mirnov coil data [15] shows that the sinusoidal portion of the waveform has an $m=3$ structure but the distorted portion has an $m \simeq 2$ structure. Strong mode distortion was not observed for PDX fishbones [33].

Figure 7 shows the radial mode structure during the early phase as measured by the ECE diagnostic. The $\delta T_{\mathrm{e}}$ fluctuations are largest near the $q=2$ radius $(R \simeq 2.1 \mathrm{~m})$. There is a $\sim 60^{\circ}$ phase variation between the weak fluctuations observed near the magnetic axis and the stronger fluctuations observed near the $q=2$ surface.

In the theory of energetic-particle instabilities, it is important to determine if the eigenfunction changes shape or remains constant during a burst. (If the eigenfunction changes shape, proper treatment of nonlinear effects requires a 'non-perturbative' model.) To investigate the temporal constancy of the mode structure, we compare the relative amplitude of the various ECE channels to the Mirnov coil as the burst evolves. For the example in figure $8(a)$, the ratio of two ECE signals remains nearly constant during the growth phase but deviates near maximum amplitude. During the growth phase, the ECE signals grow more slowly than the integrated Mirnov signal; after the maximum the ECE signals decay more slowly than $\delta B$. Figure $8(b)$ shows the average behavior for a set of similar bursts. Although the uncertainties associated with incoherent $T_{\mathrm{e}}$ fluctuations and with conditional averaging of an ensemble of bursts are substantial, the data appear to follow the same trend as the example in figure 8(a): $\delta T_{\mathrm{e}}$ gradually decreases relative to $\delta B$ during the growth phase, then rises in the decay phase. Due to the large variability of behavior in the decay phase, the standard deviations are large but examination of individual bursts shows that there are substantial differences in the evolution of the various channels during this phase. We conclude that the mode structure varies, as expected for a strongly driven non-perturbative energetic-particle mode.

The initial mode frequency is determined by the fast-ion precession frequency. Figure 9 shows calculations by an orbit-following Monte Carlo (OFMC) code [35] of the precession frequencies $f_{\text {pre }, 0}$ of fast ions deposited by the four different angles of beam injection in a typical discharge. These calculations neglect the radial electric field but analytical theory [36] and separate calculations show that inclusion of the electric field increases the actual precession 

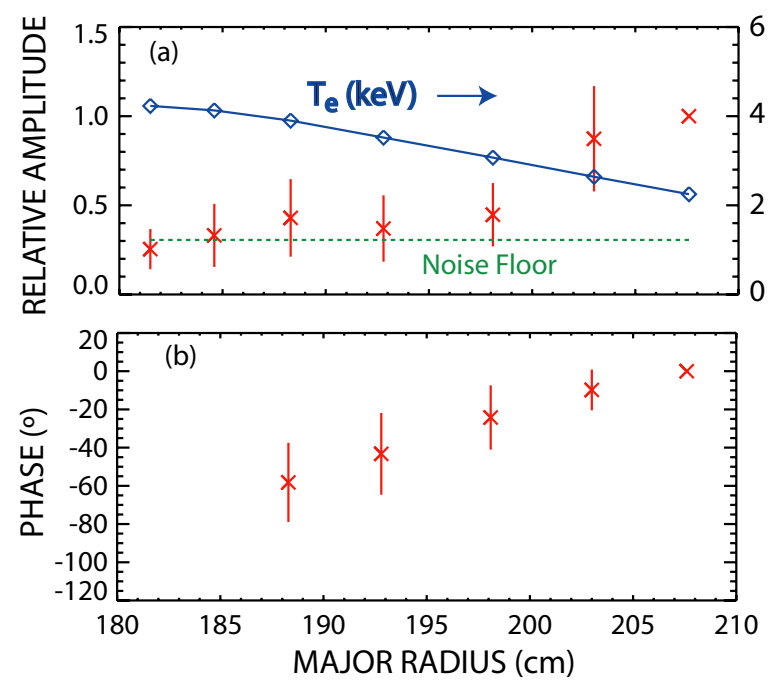

Figure 7. (a) Amplitude and ( $b$ ) phase of the mode structure early in the burst (a 1.024 ms window that ends when frequency chirping begins). The data are referenced to the outermost optically thick ECE channel at $R=208 \mathrm{~cm}$. All bursts in the database above a minimum threshold are included; the symbols represent the mean and standard deviation of the data. The dashed line indicates the estimated noise floor. The diamonds represent the electron temperature inferred from the optically thick third harmonic ECE emission. The $q=2$ surface is located between $R=208$ and $214 \mathrm{~cm}$ for most of the bursts in the database.

frequency $f_{\text {pre }}$ by an amount nearly equal to the toroidal rotation frequency $f_{\text {rot }}$, i.e.

$$
f_{\text {pre }} \simeq f_{\text {pre }, 0}+f_{\text {rot }}
$$

For both co- and counter-injection, the near-perpendicular sources produce trapped particles with $f_{\text {pre }, 0} \simeq 6 \mathrm{kHz}$ over the outer half of the plasma.

Experimentally, the initial mode frequency $f_{\mathrm{i}}$ depends on both the rotation frequency and the precession motion (figure 10). As expected, the initial frequency scales nearly linearly with $f_{\text {rot }}(r=0.64)$. The intercept of the best fit to the data is $6.8 \mathrm{kHz}$, in good agreement with calculations of the precession frequency of full-energy trapped ions deposited near the $q=2$ surface. Due to operational constraints, only modest changes in injection energy are contained in the database. Theoretically [33], the precession frequency of deeply trapped fast ions scales with $W_{\perp} / I_{\mathrm{p}}$, where $W_{\perp}$ is the perpendicular fast-ion energy. In the database, a modest dependence on this parameter is observed $(r=0.52)$ and the ratio $\Delta f_{\mathrm{i}} / \Delta f_{\mathrm{pre}, 0}$ is smaller than unity. In light of the many sources utilized, which results in a fast-ion distribution function with many different orbits, a relatively weak dependence is probably expected.

Figure 11 provides additional information about the relationship between the observed mode frequency and the fast-ion orbits. Exploiting the constancy of the magnetic moment $\mu$, the energy $W$, and the toroidal canonical angular momentum $P_{\phi}$ for collisionless orbits in the equilibrium fields, the orbit topology can be represented by contours in phase space [37,38]. In the outer portion of the plasma, stagnation and potato orbits are absent, so the relevant orbit types are the usual ones: co-passing, trapped, lost and counter-passing orbits. Figure 11 shows the orbit contours together with the fundamental precession resonance, $f_{\mathrm{i}}-f_{\text {rot }}=f_{\mathrm{pre}, 0}$, at three different radial locations. The figure also shows the birth locations of fast ions deposited by the near-perpendicular beams. Near the $q=2$ surface (middle panel), the 

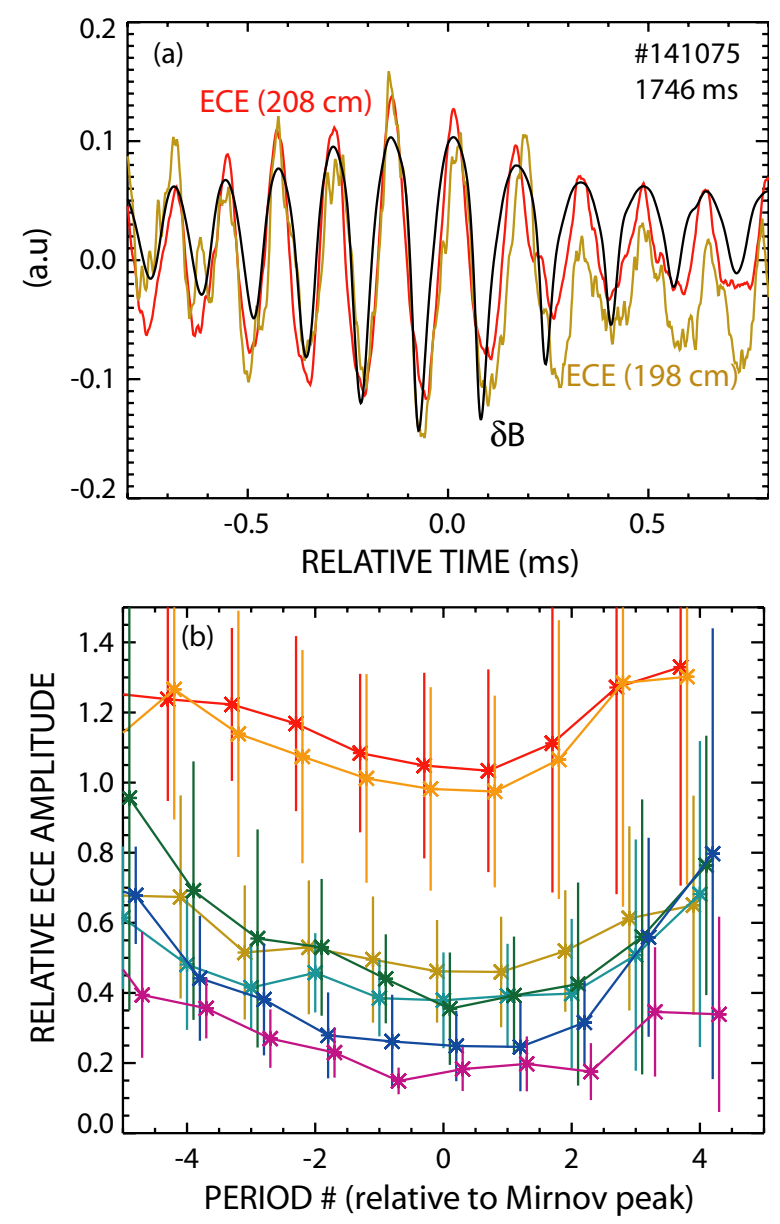

Figure 8. (a) Time evolution of two ECE channels and of the integrated Mirnov coil signal for a typical burst. The amplitudes of the signals are adjusted to facilitate comparison. $(b)$ Average ECE amplitude versus time for 16 bursts with similar peak amplitudes and growth rates. In each cycle, the ECE amplitude is normalized to the integrated Mirnov amplitude $\delta B$ in that cycle. Error bars represent standard deviations. The seven channels are the same channels in figure 7, with the amplitude monotonically increasing with radius. Low-pass filtering of the ECE data has removed some of the mode distortion.

near-perpendicular sources produce fast ions that have precession frequencies that resonate with the observed mode. Farther out in major radius (bottom panel), the resonance with fast ions produced by the counter perpendicular beam intersects a loss boundary. This intersection implies that small changes in $P_{\phi}$ induced by the mode can expel the fast ions onto loss orbits.

\subsection{Fast-ion losses}

The expected losses suggested by figure 11(c) are observed experimentally. Figure 12(a) shows an image from the FILD scintillator acquired during a fishbone burst. The gyroradius and pitch angle of the bright spot corresponds to the loss of full-energy, trapped fast ions. The spot resembles the one associated with prompt losses of the counter beams but is $\sim 2$ times brighter during the fishbone burst. Evidently, by breaking the toroidal symmetry, the $n=1$ 

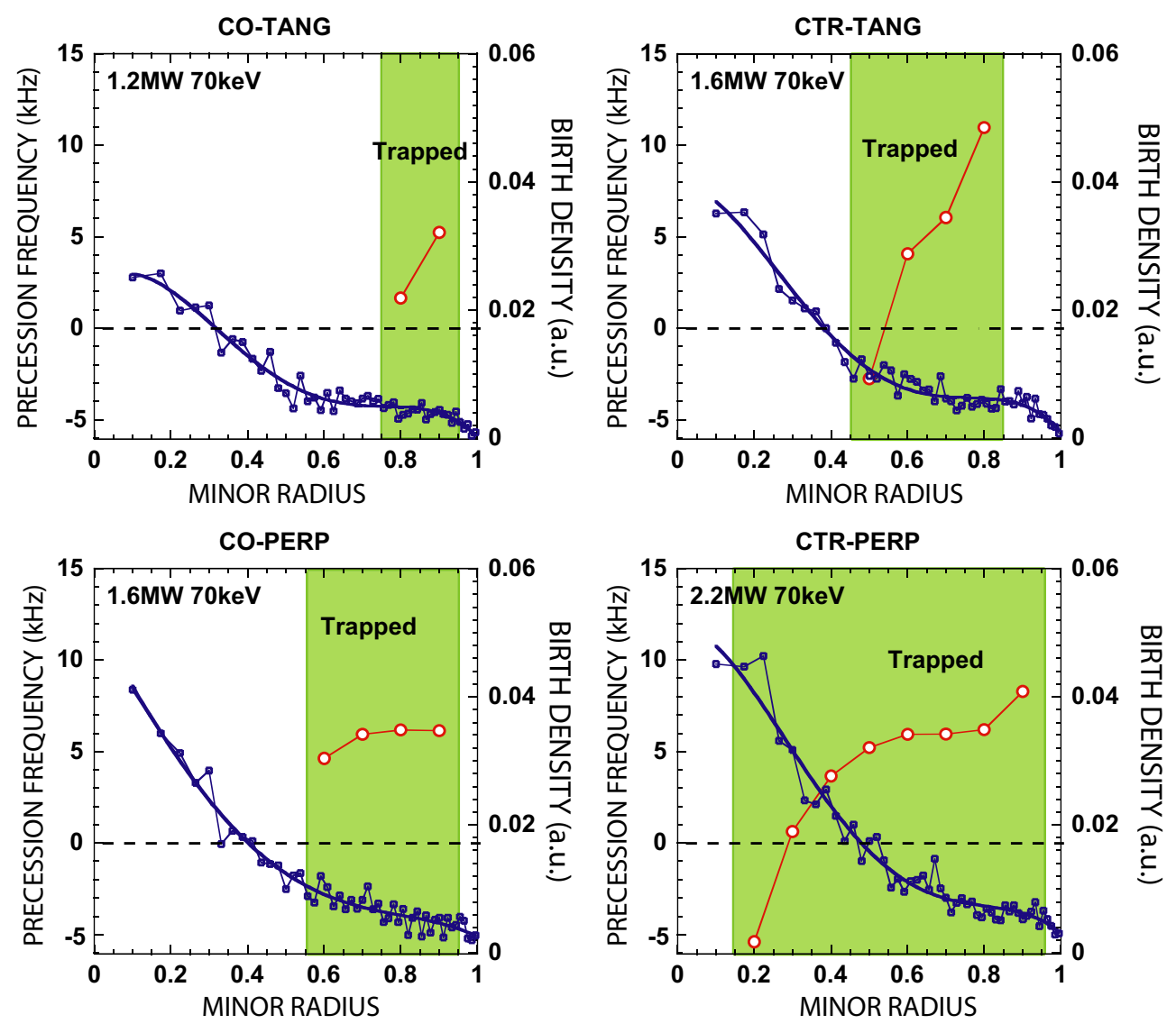

Figure 9. Calculated precession frequency (open circles-left axis) and fast-ion density at deposition (closed squares-right axis) for the four different angles of beam injection employed in the experiment. The abscissa is the normalized square root of the poloidal flux. The nearperpendicular beams create trapped orbits with precession frequencies close to the mode frequency over much of the plasma.

fishbone mode causes a decrease in $P_{\phi}$ on the inner leg of the banana that moves trapped particles from a barely confined orbit onto the loss orbit detected by FILD (figure 12(b)).

The loss of fast ions cause sudden drops in neutron emission (figure 13(a)). According to TRANSP [39] calculations, $73 \%$ of the $2.5 \mathrm{MeV}$ neutron production is from beam-target reactions, $20 \%$ are from thermonuclear reactions and $7 \%$ are from beam-beam reactions. Because the thermal deuterium density profile peaks on axis, the beam-target rate can decrease either when fast ions are expelled or when they are redistributed from the plasma core to the plasma edge; similarly, either losses or redistribution will reduce the beam-beam rate. However, since losses are actually measured, we follow the analysis of neutron data during PDX fishbones [2] and interpret the neutron rate as approximately proportional to the number of confined fast ions. For the time period shown in figure 13(a), the fishbone bursts cause an average drop in neutron rate of $9 \%$. The TRANSP code includes a 'fishbone loss' model that allows the user to expel trapped fast ions periodically. In one calculation, all trapped fast ions with energies $>50 \mathrm{keV}$ are expelled every $20 \mathrm{~ms}$. This assumption results in average drops in calculated neutron rate of $18 \%$. This proves that the trapped particle population is sufficiently 

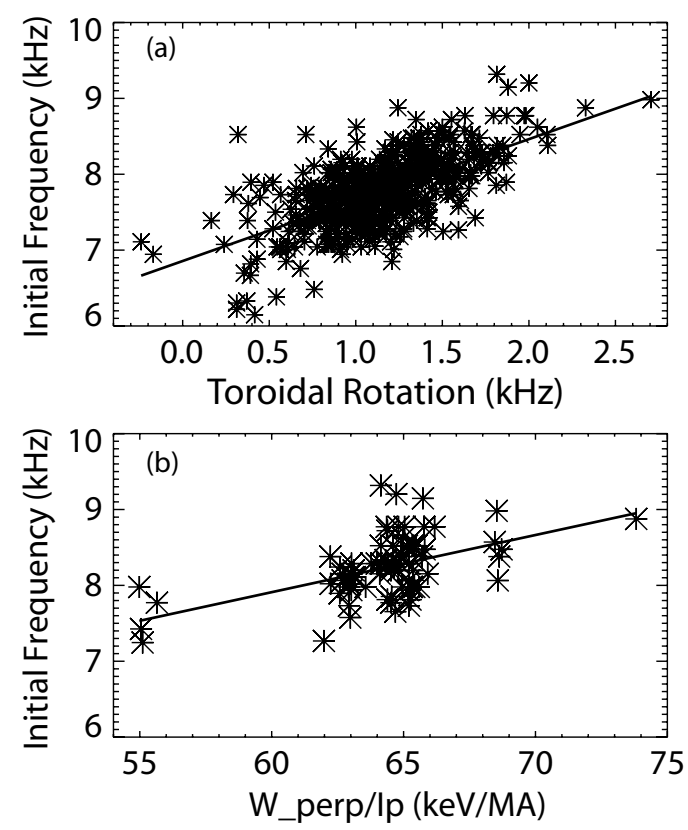

Figure 10. Initial mode frequency versus (a) toroidal rotation frequency of the $R=212 \mathrm{~cm}$ CER channel and $(b) W_{\perp} / I_{\mathrm{p}}$ for all of the discharges with valid CER data in the database. Here $W_{\perp}$ is the weighted average of the injection energies of the near-perpendicular sources and $I_{\mathrm{p}}$ is the plasma current. The lines are linear fits to the data.

large to account for all of the observed losses. In a more realistic simulation, only deeply trapped ions with energies $>50 \mathrm{keV}$ are assumed lost; this results in average calculated losses of $11 \%$. This analysis shows that a typical fishbone burst causes the loss of approximately half of the high-energy trapped fast ions.

The derivative of the neutron signal can be interpreted as an instantaneous loss rate of confined fast ions [2],

$$
v_{\text {loss }}=\dot{I}_{n} / I_{n},
$$

where $I_{n}$ is the neutron rate and $\dot{I}_{n}=\mathrm{d} I_{n} / \mathrm{d} t$. In practice, the signals are smoothed to remove high-frequency noise prior to taking the derivative and the resulting derivative is also smoothed. The maximum loss rate occurs at nearly the same time as the maximum of the Mirnov signal ( $t_{\text {neut,max }}-t_{\mathrm{Bdot} \text { max }}=-0.07 \pm 0.10 \mathrm{~ms}$ for large-amplitude bursts). The maximum loss rate also occurred at peak mode amplitude for PDX fishbones [2]. The amplitude of the peak loss rate correlates strongly ( $r=0.81$ ) with mode amplitude (figure 14(a)). A similar correlation of loss rate with mode amplitude was observed for PDX fishbones [2]. Theoretically, the dependence of the loss rate on mode amplitude can provide information on the mechanism of fast-ion transport, with a linear dependence often being associated with convective transport, while a quadratic dependence is often associated with diffusion. A quadratic dependence provides a slightly better fit to these neutron data than a linear fit $\left(20 \%\right.$ smaller $\left.\chi^{2}\right)$. Since the frequency chirp rate also correlates with mode amplitude, the peak loss rate correlates $(r=0.71)$ with the magnitude of the frequency chirp as well (figure $14(b)$ ).

All seven loss diagnostics detect bursts in conjunction with the fishbone activity (figure 15). (The various diagnostics were not all available on the same discharges, so samples of the best available data from each diagnostic are displayed.) The BILD detector usually saturated. 

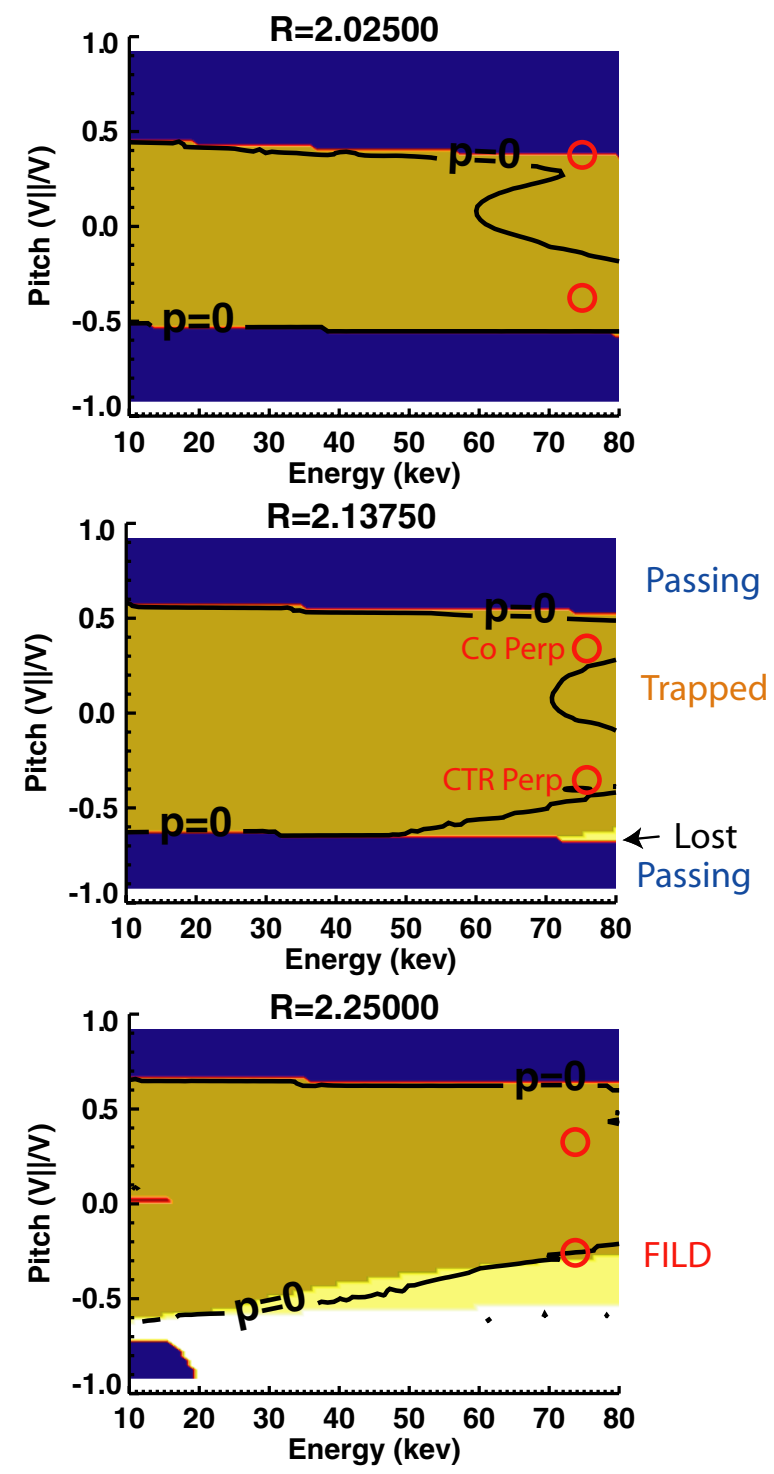

Figure 11. Orbit topology maps at the midplane for three different radial locations for a typical equilibrium. The contours are calculated by a constants-of-motion code [38]. The abscissa is the fast-ion energy and the ordinate is the pitch relative to the magnetic field, $v_{\|} / v$. (Positive values travel in the co-current direction.) The central regions are occupied by trapped particles, while passing particles are at large values of $\left|v_{\|} / v\right|$. A loss boundary exists for high-energy, counter-going ions (near $v_{\|} / v=-0.5$ ). The lines indicate regions that satisfy the fundamental precession-frequency resonance, $f_{\mathrm{i}}-f_{\mathrm{rot}}=f_{\mathrm{pre}, 0}$. The circles represent the approximate center of beam deposition for the co-perpendicular and counter-perpendicular beams. The loss orbit detected by the FILD diagnostic (bottom panel) occurs near the loss boundary for orbits deposited by the counter-perpendicular beam and also coincides with the precession-frequency resonance.

Figure 15(a) shows an example from a relatively weak burst that did not saturate the electronics. As shown in this example, the largest BILD signals typically occur $\sim 0.5 \mathrm{~ms}$ after the peak Mirnov amplitude. Except in a few cases with very large decay rates (and then only during the decay phase), the BILD burst always occurs at the same phase relative to the mode. This 

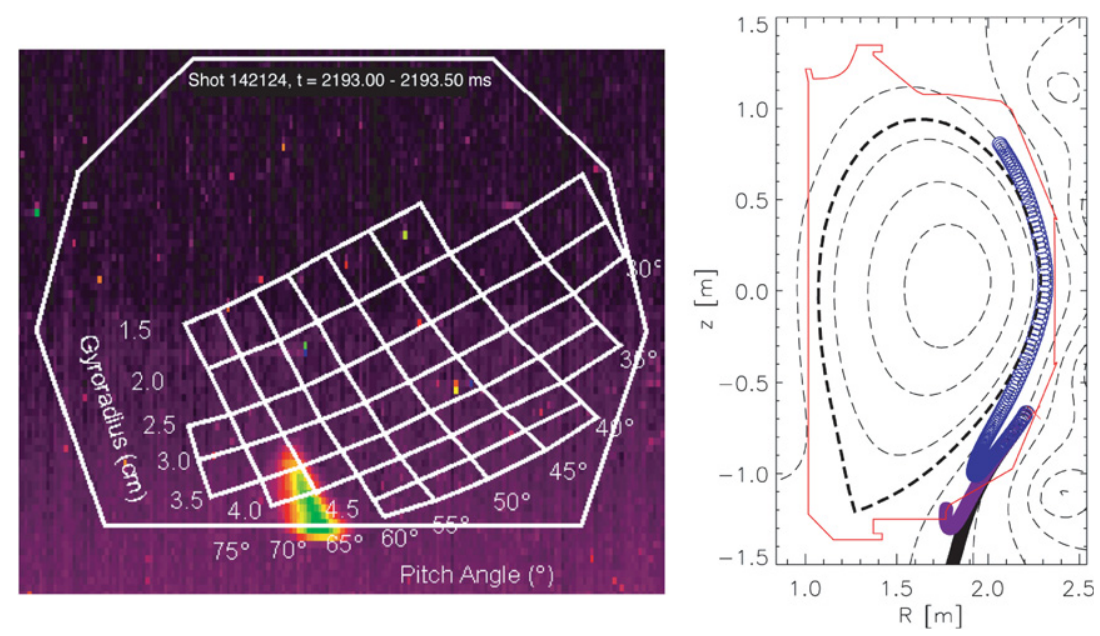

Figure 12. (a) Bright spot produced by ejected trapped particles on the FILD scintillator during fishbone activity. The grid relates locations on the scintillator to the gyroradius and pitch angle of lost ions. (b) Trapped particle orbit that strikes the scintillator at the bright spot (calculated in the equilibrium field).

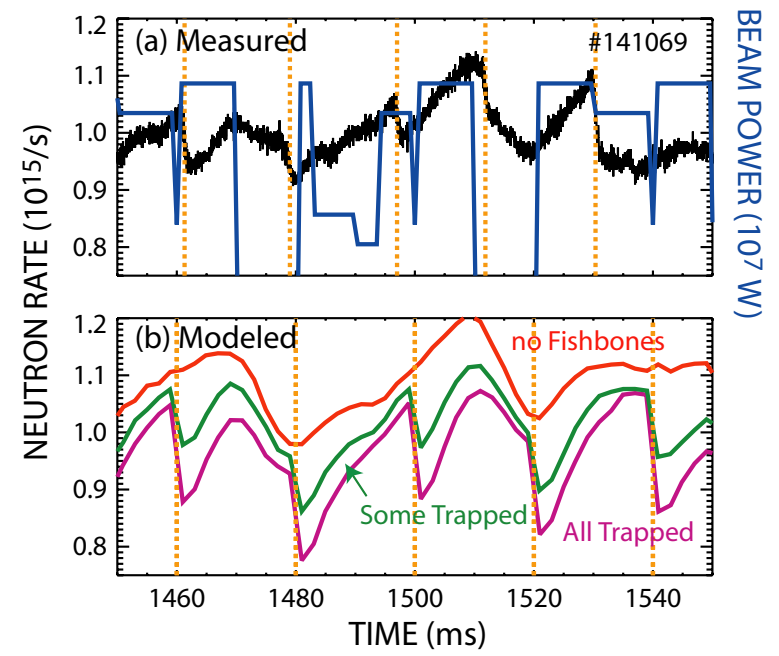

Figure 13. (a) Time evolution of the neutron rate and beam power in a discharge with regular fishbone bursts (indicated by the dashed vertical lines). The beam power is modulated to maintain nearly constant normalized beta. (b) TRANSP modeling of the same discharge. The upper trace neglects the fishbones. The lower trace ejects all trapped ions with $E>50 \mathrm{keV}$ every $20 \mathrm{~ms}$, while the intermediate trace only ejects deeply trapped ions with $E>50 \mathrm{keV}$.

type of 'beacon' of lost particles with a fixed phase relative to the mode was also observed for PDX fishbones [3]. This behavior is predicted by the mode-particle pumping theory of convective transport that was used to explain transport by PDX fishbones [5]. The fast ions only experience an outward $\boldsymbol{E} \times \boldsymbol{B}$ drift for a given phase of $\boldsymbol{E}$, so a given diagnostic always observes expelled particles at the same phase relative to the mode.

The reciprocating Langmuir probe also observes a beacon of expelled fast ions (figure $15(b)$ ). For this observation, the probe was carefully positioned $6.1 \mathrm{~cm}$ from the 


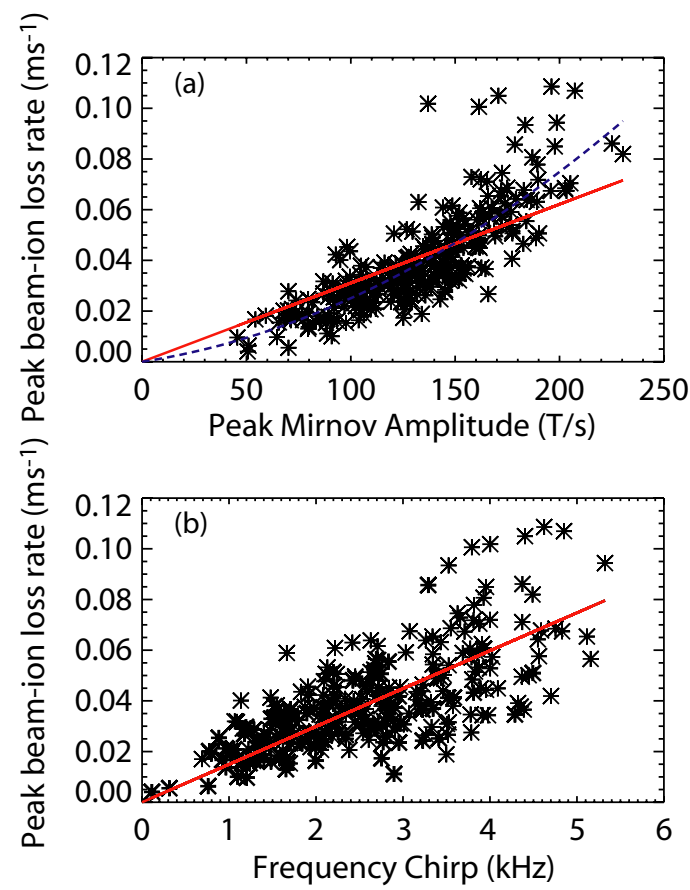

Figure 14. Maximum neutron loss rate versus $(a)$ maximum Mirnov amplitude and $(b)$ change in mode frequency, $f_{\mathrm{i}}-f_{\text {final }}$ for all of the bursts in the database with constant beam power during the burst. Linear (solid lines) and quadratic (dashed line) fits to the data are included.

last-closed flux surface and $5.6 \mathrm{~cm}$ from the vessel wall. This location minimizes contributions to the ion saturation current from thermal ions, while still allowing fast ions to strike the collector tip.

High-bandwidth FILD measurements became available at the end of the experimental campaign. For this measurement, the photomultiplier imaged a portion of the scintillator centered on a lower pitch angle $\left(\sim 50^{\circ}\right)$ than the primary fishbone loss peak at $\sim 70^{\circ}$. Nevertheless, evidence of a coherent beacon is also observed by this diagnostic (figure $15(c)$ ).

Two NPA channels made high-bandwidth measurements throughout the campaign. The NPA sightlines originate from a lower port, rise up to intersect the counter beams near the midplane, then strike the inner wall above the midplane; toroidally, the sightlines are normal to the plasma. Whether the viewed counter beams are off or on, fishbone modes cause beaconlike bursts on the NPA detectors (figure 15(c)). Presumably, these bursts are caused by fast ions that are expelled to the high neutral density region at large major radius. On PDX, the signal on the vertically viewing NPA that viewed the outer edge of the plasma was two orders of magnitude larger than the NPA that viewed the inner edge of the plasma [3,40]. There also are some indications of reductions in active signal caused by the fishbone burst.

Normally, the signal for the BES diagnostic is dominated by beam emission from the active injected beam but, in the case of large losses of fast ions, FIDA light from the edge plasma can also be appreciable [30]. In DIII-D, the BES filters accept Doppler-shifted D-alpha light produced by fast ions on escaping trapped orbits. To avoid the complication of concurrent beam emission, the data in figure $15(d)$ are from a discharge where the active beams were both off. Bursts of light are observed. In the case of the BES diagnostic, two bursts per cycle are often observed because the BES sightline intercepts the plasma edge at two different toroidal 

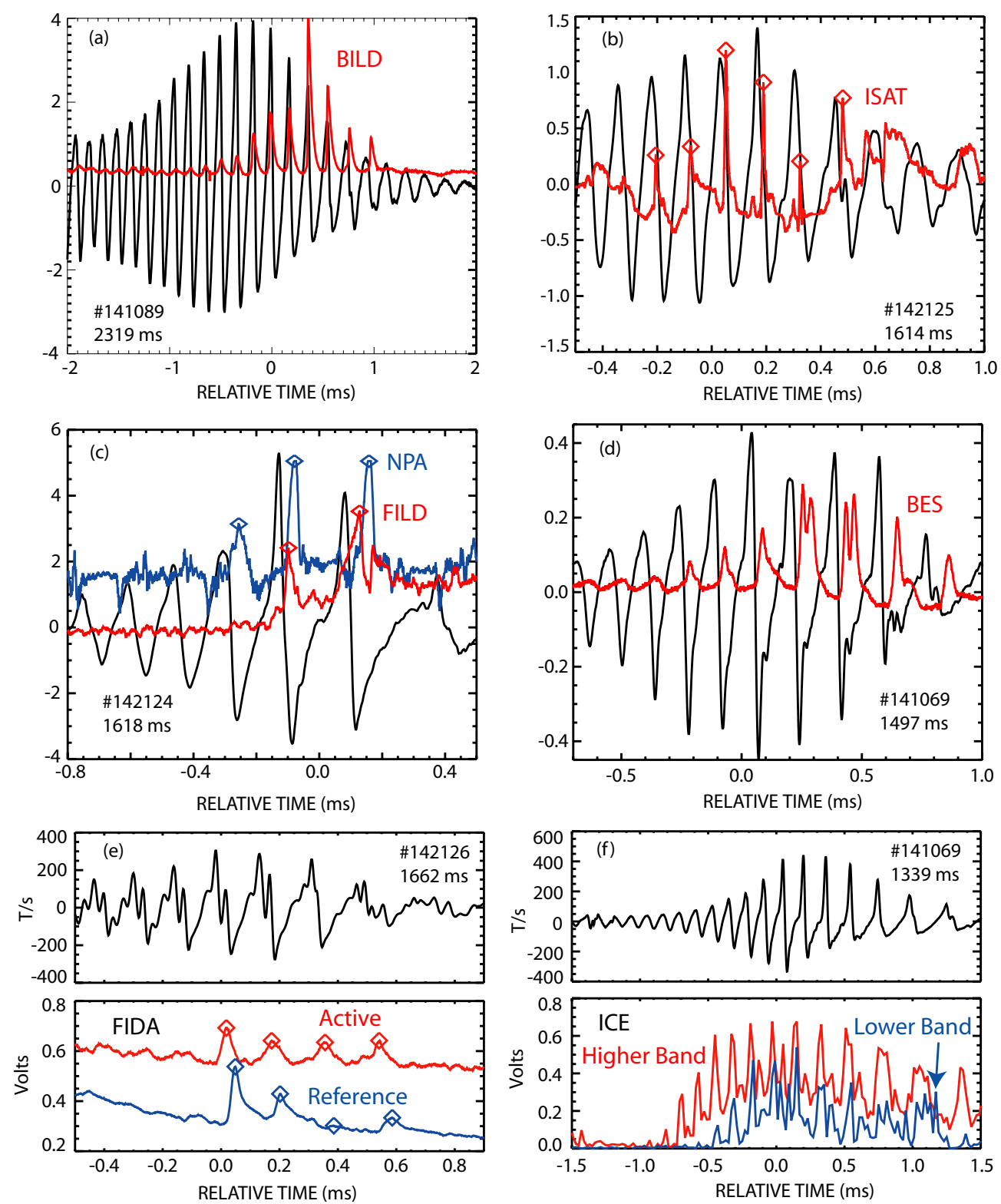

Figure 15. Loss-detector data during a fishbone burst from $(a)$ the BILD foil, $(b)$ the Langmuir probe $I_{\text {sat }}$ tip, $(c)$ the FILD scintillator and SSNPA detector, $(d)$ a central BES channel on a discharge without any beam emission, $(e)$ active and passive f-FIDA channels and $(f)$ ICE passband signals that include the fundamental cyclotron resonance (lower band) or the first few harmonics (higher band). The diamonds represent peaks used to measure the phase relative to the Mirnov signal.

locations. The sightline is angled slightly downward. The first peak corresponds to a poloidal position below the midplane, while the second peak corresponds to a poloidal position above the midplane. Generally, the first peak (lower poloidal position-in the direction of the $\nabla B$ drift) appears earlier in the evolution of the mode and is larger in amplitude than the second peak, although often the two peaks are comparable during the decay phase. Like the other edge diagnostics, both peaks have a fixed phase relative to the mode. 


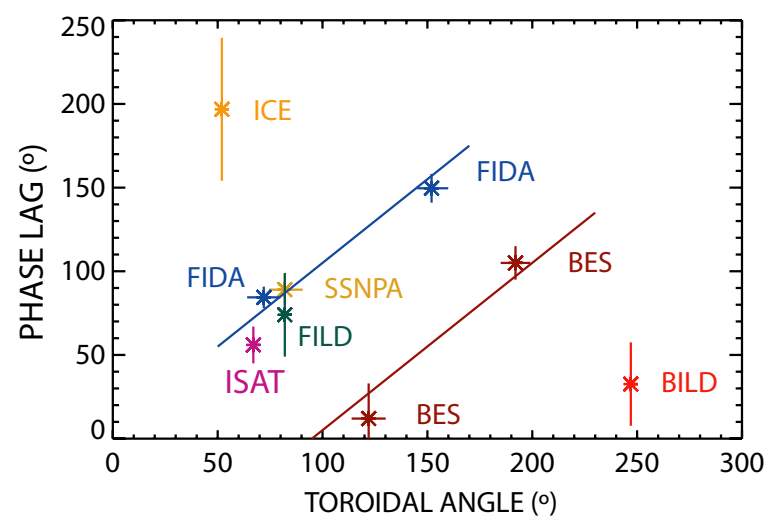

Figure 16. Measured phase lag relative to the Mirnov signal versus toroidal angle of the diagnostic. The vertical error bar represents the standard deviation over several cycles and the horizontal error bar is an estimate of the uncertainty in angle. The solid lines show the expected variation for an $n=1$ mode

Bursts of edge FIDA light are also observed by a dedicated FIDA diagnostic (figure 15(e)). The sightlines for this diagnostic look down at a canted toroidal angle from an upper port toward the vessel floor [28]. One channel views one toroidal direction to intersect a heating beam (for active FIDA measurements), while the other channel views in the opposite toroidal direction to provide a reference signal. The bandpass filter for these measurements accepts Doppler-shifted light from co-going ions in the case of the active view and from counter-going ions for the reference view. Both sightlines intersect the edge region near the top and bottom of the vessel. Both channels observe beacon-like bursts of signal that presumably are caused by expulsion of fast ions by the fishbones. Because the $\nabla B$ drift is downward, the bursts are probably caused by fast ions that charge exchange with neutrals near the bottom of the vessel.

Beacon-like bursts of signal are also measured by the ICE diagnostic (figure 15( $f$ )). Both the higher band and lower band filtered signals measure bursts with a definite phase relative to the mode, although the variation of the phase is somewhat larger than for the other loss diagnostics. The higher band signal correlates more reliably with the Mirnov signal than the lower band signal. For the higher band, like the maximum neutron loss rate, the signal peaks at the same time as the maximum Mirnov amplitude (within statistical error). The magnitude of the ICE signal summed over the burst also correlates strongly with the maximum mode amplitude $(r=0.52)$. Given that the ICE radiation is produced through a two-stage process (the fishbones expel fast ions, which then excite magnetoacoustic waves), it is rather surprising that the ICE signal provides such a reliable measurement of the beacon and of the timing and magnitude of the losses.

The phase of all of the beacon measurements is summarized in figure 16. For diagnostics that measure the same quantity, such as the two FIDA measurements and the two BES peaks, the phase difference between the measurements agrees with the toroidal separation of the detection points, as expected for an $n=1$ mode. For the larger dataset, although each diagnostic measures a well-defined phase relative to the mode, there is no clear pattern for the entire set of measurements. Upon reflection, this is not surprising. The fishbone mode rotates at the relatively slow precession frequency. On the other hand, the phase of any particular diagnostic signal depends on the final leg of the orbit of the fast ions that produce that signal; this final leg occurs at the much higher bounce frequency. On this final leg, a loss orbit such as the one shown in figure $12(b)$ moves over $90^{\circ}$ toroidally. Since the different diagnostics 

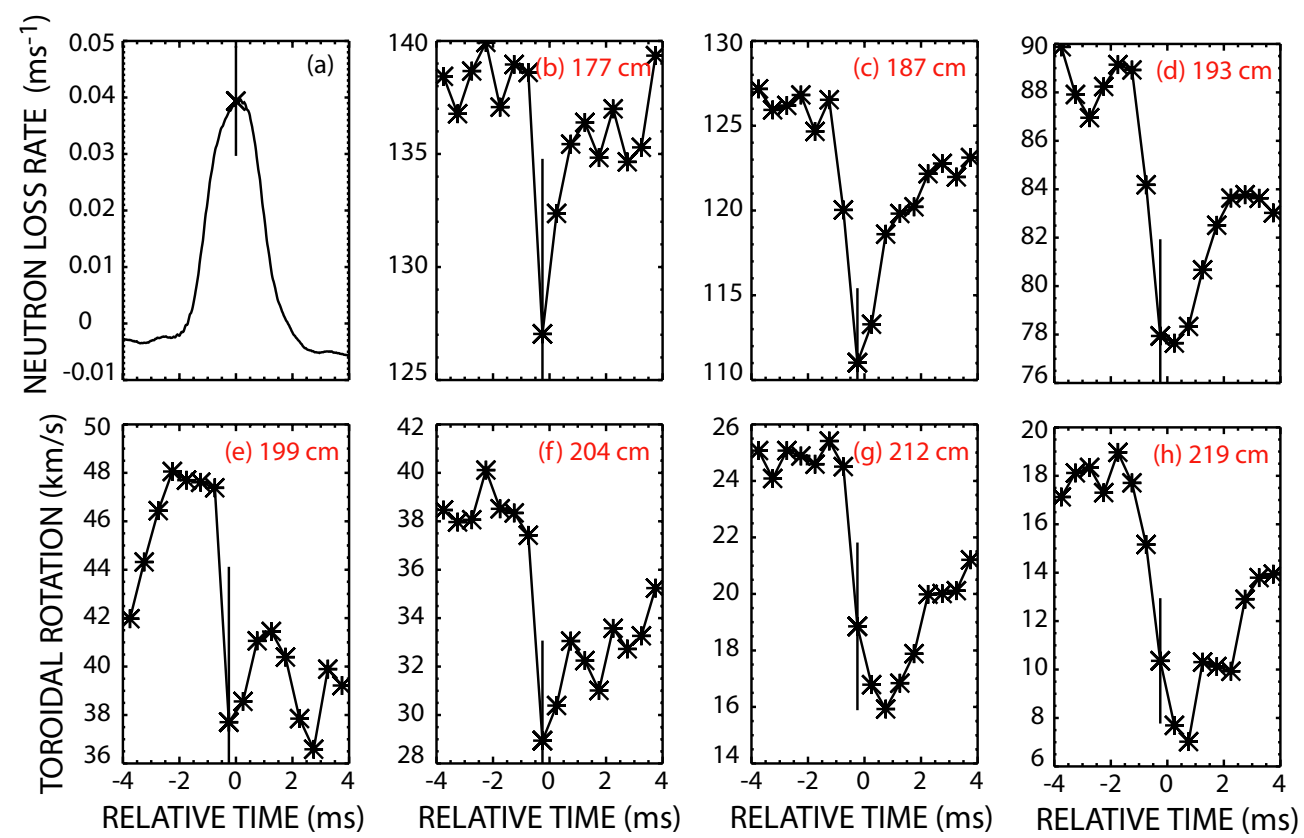

Figure 17. Time evolution of $(a)$ the fast-ion loss rate inferred from the drop in neutron signal and $(b)-(h)$ the toroidal rotation for 7 CER channels. The data are conditionally averaged over an ensemble of relatively large $\left(130<B_{\max }<180 \mathrm{~T} \mathrm{~s}^{-1}\right)$ fishbone bursts. The error bar in $(a)$ represents the standard deviation of the peak loss rate. The error bars in $(b)-(h)$ represent the standard deviation of the relative change between -0.75 and $-0.25 \mathrm{~ms}$.

measure different types of loss orbits, the final orbit leg effectively scrambles the toroidal phase of the various measurements. A similar result was obtained for PDX fishbones. Both the NPA [3] and a silicon detector that directly measured lost fast ions [41] observed a beacon but the two diagnostics did not observe the same phase relative to the mode [33].

Since the fast-ion losses are non-ambipolar, they should transiently alter the radial electric field $E_{r}$. Because the plasma must preserve radial force balance, a changing electric field alters the toroidal rotation. To detect this effect, CER measurements of toroidal rotation are acquired in $0.5 \mathrm{~ms}$ time bins. To obtain adequate statistics, data from 16 similar fishbone bursts are conditionally averaged. The results appear in figure 17. All CER channels measure an abrupt drop in toroidal rotation of $\sim-10 \mathrm{~km} \mathrm{~s}^{-1}(\lesssim 1 \mathrm{kHz})$ when the neutron loss rate is greatest. This suggests that fast-ion losses occur throughout the plasma. Comparing channels, the drop persists longer at large major radius than it does in the core. The magnitude of the drop is comparable to the $\sim+10 \mathrm{~km} \mathrm{~s}^{-1}$ jump in toroidal rotation that is observed when a single neutral beam source injects for $10 \mathrm{~ms}$ [42]. Evidently, the fishbone burst acts like a 'negative beam blip.' A $10 \mathrm{~ms}$ beam blip injects about $+0.5 \mathrm{C}$ of charge. From the $\sim 10 \%$ drop in neutron rate for these bursts, the estimated charge of expelled fast ions is $\sim-0.3 \mathrm{C}$, so the "negative beam blip' associated with the fishbone should reduce the toroidal rotation about as much as a positive beam blip increases it, just as is observed.

\subsection{Confined fast ions}

In addition to the drop in signal associated with fast-ion losses, the neutron signal also contains oscillations (figure 18). After filtering out high-frequency noise, these oscillations are visible 

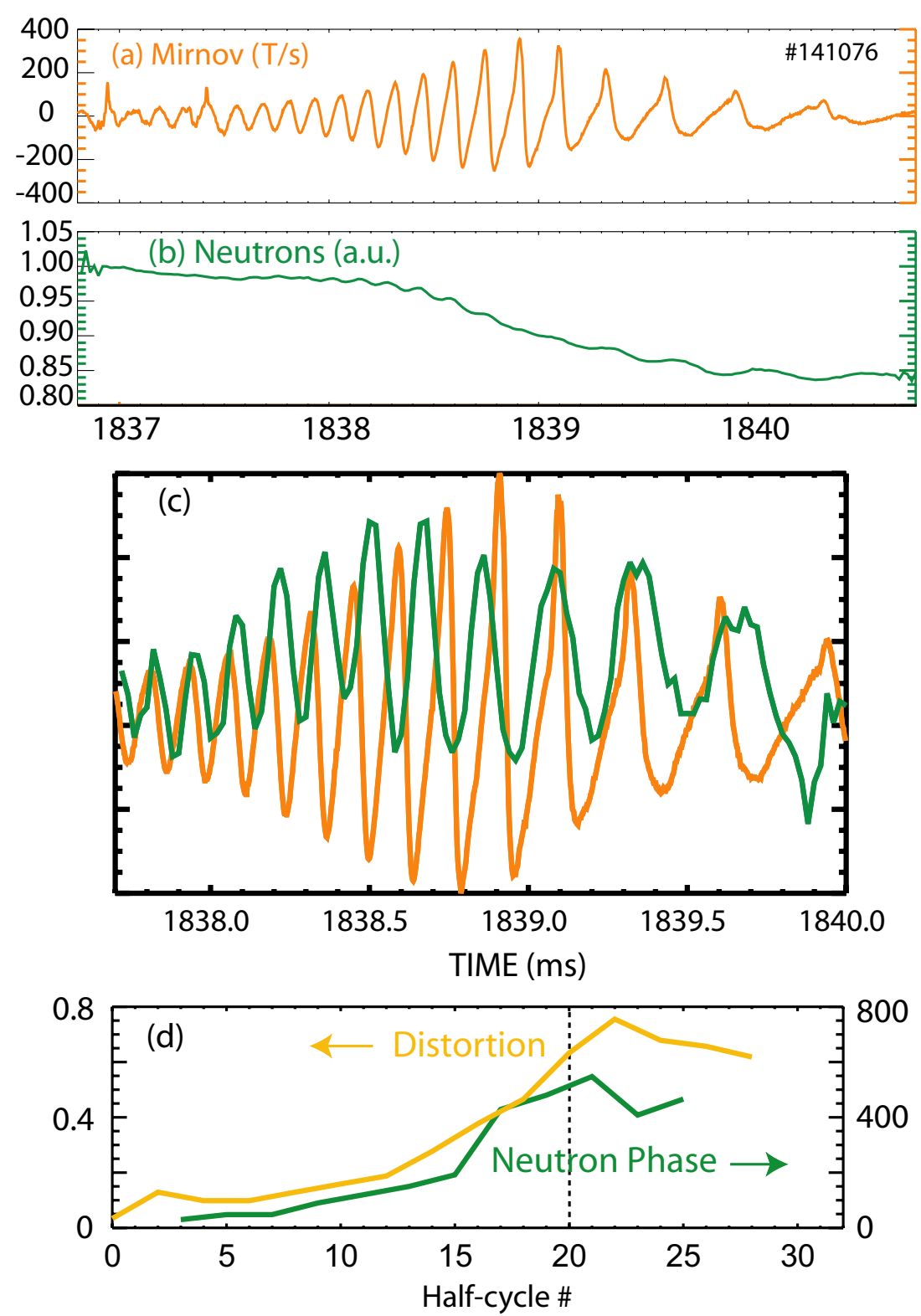

Figure 18. Time evolution of $(a)$ the Mirnov coil signal and $(b)$ the neutron signal during a fishbone burst. ( $c$ ) Overlay of the oscillations in the Mirnov signal and the neutron signal. An error function fit to the drop in the neutron rate is used to detrend the neutron signal. $(d)$ Evolution of the mode distortion and neutron phase (degrees) versus half-period number. The dashed vertical line indicates the time of maximum amplitude.

in the total signal (figure 18(b)) but they become particularly evident after detrending the signal to remove the overall drop (figure 18(c)). In contrast to all of the lost-ion measurements, the phase of these neutron oscillations does not remain constant throughout the fishbone burst. Instead, the phase steadily slips relative to the Mirnov trace as the burst evolves (figure 18(c)). The phase slippage is largest near maximum amplitude, which is also when the mode distortion 

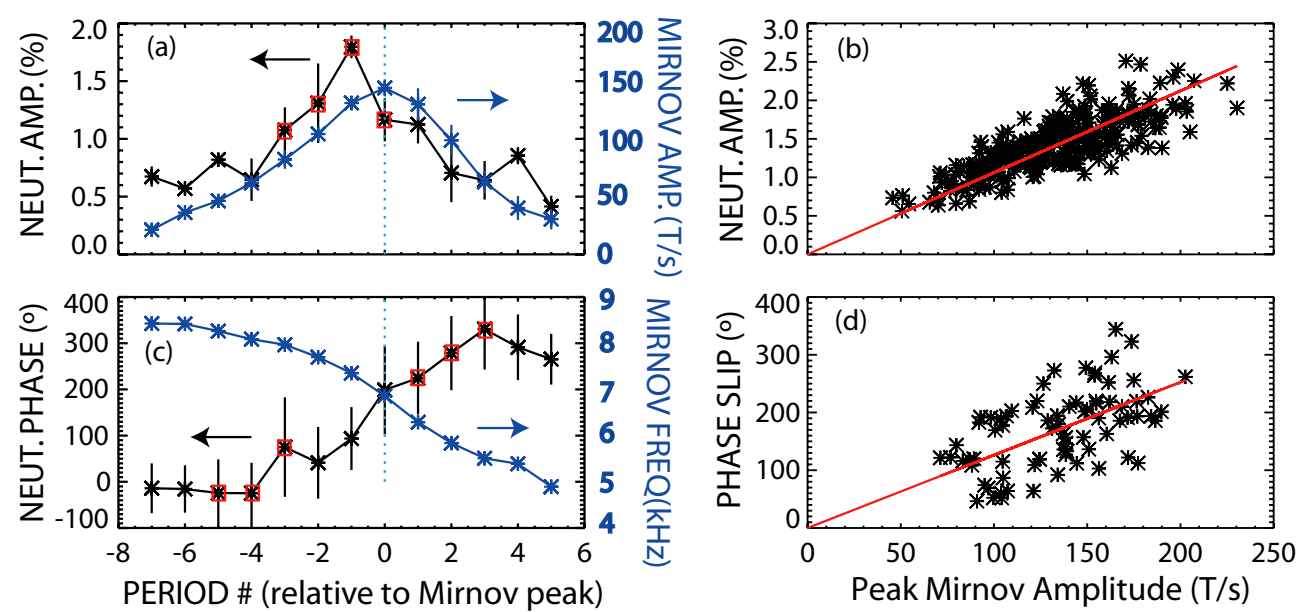

Figure 19. Average neutron fluctuation $(a)$ amplitude and $(c)$ phase versus period number for 40 relatively large $\left(140<B_{\max }<162 \mathrm{~T} \mathrm{~s}^{-1}\right)$ fishbone bursts. The evolution of the mode $(a)$ amplitude and $(c)$ frequency for the same bursts is also shown. The error bars represent the standard deviation for this ensemble. (b) Neutron fluctuation amplitude and $(d)$ phase slippage versus maximum Mirnov amplitude for all bursts with constant beam power during the burst. The lines are linear fits to the data. The amplitude is an average over cycles $-3-0$; the phase slippage is the average over cycles $-5-3$ subtracted from the average over cycles $1-3$; the utilized cycles are highlighted by boxes in $(a)$ and $(c)$, respectively.

and frequency chirping rapidly increase (figure $18(d)$ ). For this particular case, in the course of the burst, the phase slips over $360^{\circ}$.

Because it measures (predominately) virgin neutrons, the plastic scintillator signal is sensitive to the geometrical position of the confined fast ions that produce the neutron reactions. Neutron oscillations of similar amplitude were measured for PDX fishbones [2]. Strachan et al [2] showed that these oscillations were caused by the helical distortion of the neutronemitting core. Using the expected geometrical efficiency of the plastic scintillator and the measured displacement of the internal kink mode, they showed that the predicted oscillations agree in both amplitude and phase with the observations. No phase slippage was reported.

Strachan's model cannot account for the phase slippage associated with off-axis fishbones. Presumably, as for PDX fishbones, the oscillations are associated with motion of confined fast ions toward and away from the detector. (Motion relative to the thermal deuterium density gradient may also contribute.) But the phase slippage indicates that the oscillations of the majority of confined fast ions decouples from the mode itself, even while the expelled ions retain their phase. This decoupling accelerates when the mode is large, distorting and changing rapidly in frequency.

This phenomenon occurs for all off-axis fishbone bursts. Figures 19(a) and $(c)$ show the average behavior for a set of similar bursts. The neutron fluctuation amplitude usually peaks slightly before maximum Mirnov amplitude. (For an average of all of the bursts in the database, the peak occurs two cycles prior to the Mirnov maximum.) The phase changes most rapidly when the frequency changes. Note that a fixed temporal delay relative to the mode would produce phase changes of the opposite sign. For the entire database, the neutron fluctuation amplitude scales linearly with maximum Mirnov amplitude (figure 19(b)) $(r=0.80)$. The phase slippage also tends to increase with maximum Mirnov amplitude (figure 19(d)) $(r=0.55)$. In this latter case, the relatively large uncertainty in determination of the phase slip may account for some of the scatter. 


\section{Discussion and conclusion}

Many features of DIII-D off-axis fishbones are similar to PDX $q=1$ fishbones.

- The modes occur in high-beta plasmas near an MHD stability limit.

- They are $n=1$ modes driven by neutral beam ions.

- The initial mode frequency matches the precession frequency of full-energy trapped ions; also, the radial profile of the precession frequency is fairly flat, so fast ions can stay in resonance while being transported radially.

- The frequency chirp is large $\left(\Delta f / f_{\mathrm{i}} \simeq 0.4\right)$ and increases with increasing mode amplitude. Also, during a burst, the frequency chirp rate is largest near maximum mode amplitude.

- The growth rate is $\gamma / \omega \simeq 5 \%$.

- The modes appear in repetitive bursts. (The burst cycle was more regular for PDX fishbones but this difference is probably caused by the strong beam modulation in the DIII-D experiments.)

- The modes cause losses of fast ions that peak near the time of maximum mode amplitude.

- The magnitude of the losses scales approximately linearly with mode amplitude.

- Fast ions are expelled to the outside of the plasma in a 'beacon' (with a fixed phase relative to the mode).

- Higher $n$ modes with similar phenomenology are occasionally observed, as they were on PDX [2].

In addition, the loss of fast ions causes a sudden drop in toroidal rotation. This effect was not measured on PDX but probably occurred.

On the other hand, several features of DIII-D off-axis fishbones differ from PDX fishbones.

- On PDX, the decay rate was smaller than the growth rate but the opposite is true for off-axis fishbones. Also, the decay phase is highly variable for off-axis fishbones but, in PDX, except when a sawtooth crash occurred, the decay rate was regular.

- On PDX, the waveform remained nearly sinusoidal throughout the burst. In contrast, for off-axis fishbones, the waveform always becomes highly distorted near maximum mode amplitude.

- On PDX, the fluctuations in the neutron signal maintained a fixed phase relative to the mode. For off-axis fishbones, the neutron phase slips continuously when the amplitude is large and the mode frequency and waveform shape are changing rapidly.

In addition, for off-axis fishbones, the radial eigenfunction changes shape during the evolution of a burst. This information is not available for PDX fishbones.

The many similarities between PDX fishbones and off-axis fishbones supports the notion advanced by Okabayashi et al [15] that off-axis fishbones are an energetic-particle branch of a kink mode, just as PDX fishbones are an energetic-particle branch of the $1 / 1$ internal kink. In this paradigm, the burst cycle resembles classic predator-prey relaxation oscillations because the increase in fast-ion density associated with beam fueling pushes a marginally stable mode across the stability boundary, then the expulsion of the fast ions stabilizes the mode $[6,43]$. In contrast to a resistive wall mode (or other normal mode of the background plasma), the mode frequency is determined by the precession frequency of the fast ions. The large frequency chirping and the non-perturbative nature of the eigenfunction are consistent with the idea that the off-axis fishbone is a beam mode that exists due to the intense fast-ion population.

What accounts for the differences in nonlinear behavior of PDX and off-axis fishbones? One possible explanation is that the off-axis fishbone is an external mode instead of an internal mode. (The response of the instability to feedback is strong evidence for the external nature of 
the off-axis fishbones [15].) In this hypothesis, the mode distortion is caused by the dissipation associated with the conducting wall. Another difference between PDX fishbones and off-axis fishbones is the beam fueling. In PDX, all of the sources injected at the same angle, so the fast-ion distribution function was concentrated at a single pitch angle. In contrast, the DIII-D experiments employ many angles of injection. (In addition, $T_{\mathrm{e}}$ is sufficiently high that the pitch-angle scattering rate is comparable to the slowing-down rate, which further isotropizes the distribution function.) Perhaps the response of confined fast ions from other sources is responsible for the phase slippage in the neutron oscillations and also contributes to the distortion of the mode.

A key goal of the energetic-particle community is to develop a predictive capability for fast-ion driven instabilities. The fascinating nonlinear evolution of off-axis fishbones is a ripe topic for theoretical study.

\section{Acknowledgments}

We thank John deGrassie, Boris Breizman, Liu Chen, and the referees for helpful insights and gratefully acknowledge the invaluable contributions of the DIII-D team. This work was funded by the US Department of Energy under SC-G903402 and DE-FC02-04ER54698 DE-FG02-07ER54917.

\section{References}

[1] Mcguire K et al 1983 Phys. Rev. Lett. 50891

[2] Strachan J D et al 1985 Nucl. Fusion 25863

[3] Beiersdorfer P, Kaita R and Goldston R J 1984 Nucl. Fusion 24487

[4] Goldston R J et al 1987 Nucl. Fusion 27921

[5] White R B et al 1983 Phys. Fluids 262958

[6] Chen L, White R B and Rosenbluth M N 1984 Phys. Rev. Lett. 521122

[7] Heidbrink W W and Sadler G J 1994 Nucl. Fusion 34535

[8] Coppi B and Porcelli F 1986 Phys. Rev. Lett. 572272

[9] Chen L 1994 Phys. Plasma 11519

[10] Porcelli F 1991 Plasma Phys. Control. Fusion 331601

[11] Huysmans G T A et al 1999 Nucl. Fusion 391489

[12] Matsunaga G et al 2009 Phys. Rev. Lett. 103045001

[13] Matsunaga G et al 2010 Nucl. Fusion 50084003

[14] Okabayashi M et al 2009 Nucl. Fusion 49125003

[15] Okabayashi M et al 2011 Phys. Plasma 18056112

[16] Helander P, Gimblett C G, Hastie R J and Mcclements K G 1997 Phys. Plasma 42181

[17] Freidberg J P 1987 Ideal Magnetohydrodynamics (New York: Plenum) section 9.4.6

[18] Lao L L, St John H, Stambaugh R D, Kellman A G and Pfeiffer W 1985 Nucl. Fusion 251611

[19] Rice B W, Nilson D G and Wroblewski D 1995 Rev. Sci. Instrum. 66373

[20] Strait E J 2006 Rev. Sci. Instrum. 77023502

[21] Austin M E and Lohr J 2003 Rev. Sci. Instrum. 741457

[22] Gohil P, Burrell K H, Groebner R J and Seraydarian R P 1990 Rev. Sci. Instrum. 612949

[23] Heidbrink W W 1986 Rev. Sci. Instrum. 571769

[24] Zhu Y B, Heidbrink W W and Pickering L D 2010 Nucl. Fusion 50084024

[25] Fisher R K et al 2010 Rev. Sci. Instrum. 81 10D307

[26] Watkins J G et al 1992 Rev. Sci. Instrum. 634728

[27] Luo Y, Heidbrink W W, Burrell K H, Gohil P and Kaplan D 2007 Rev. Sci. Instrum. 78033505

[28] Muscatello C M, Heidbrink W W, Taussig D and Burrell K H 2010 Rev. Sci. Instrum. 81 10D316

[29] Gupta D K, Fonck R J, Mckee G R, Schlossberg D J and Shafer M W 2004 Rev. Sci. Instrum. 753493

[30] Heidbrink W W, Mckee G R, Smith D R and Bortolon A 2011 Plasma Phys. Control. Fusion 53085007

[31] Gorelenkov N N and Cheng C Z 1995 Nucl. Fusion 351743

[32] Watson G W and Heidbrink W W 2003 Rev. Sci. Instrum. 741605 
[33] Buchenauer D A J 1985 Fast ion effects on magnetic instabilities in the PDX tokamak PhD Thesis Princeton University

[34] Heidbrink W W 1995 Plasma Phys. Control. Fusion 37937

[35] Shinohara K et al 2007 Nucl. Fusion 47997

[36] Graves J P, Hastie R J and Hopcraft K I 2000 Plasma Phys. Control. Fusion 421049

[37] White R B 2001 The Theory of Toroidally Confined Plasmas 2nd edn (London: Imperial College Press)

[38] Van Zeeland M A et al 2011 Phys. Plasma 18056114

[39] Budny R V 1994 Nucl. Fusion 341247 and references therein

[40] Heidbrink W W and Beiersdorfer P 1987 Nucl. Fusion 27608

[41] Buchenauer D, Heidbrink W W, Roquemore L and Mcguire K 1987 Rev. Sci. Instrum. 582264

[42] Degrassie J S, Groebner R J and Burrell K H 2006 Phys. Plasma 13112507

[43] Heidbrink W W et al 1993 Phys. Fluids B 52176 